\title{
Design of Electric Vehicle Drive Motor ECU Control System Based on AUTOSAR
}

\author{
Zhiyu Huang ${ }^{a}$, Zhangliang Xiong ${ }^{b}$ \\ Embedded engineering center of automotive electronics, Chongqing University of Posts, Chongqing \\ 400065, China \\ ahuangzy@cqupt.edu.cn, bzhangliang198763@163.com
}

Keywords: PMSM; ECU; AUTOSAR; Software Components; Runnable Entities; VFB/RTE; BSW.

\begin{abstract}
In view of the problem of the Electric vehicle, which is in difficulty and reusability in the development with the permanent magnet motor (PMSM) ECU control system, this paper presented a ECU motor control system development method based on automotive open system architecture (AUTOSAR). It mainly to complete the design of the application layer software components, runnable entities, VFB/RTE layer and basic software layer according to AUTOSAR hierarchical, modularization idea. Bench experiment results showed that the motor ECU control system based on the standard of AUTOSAR, implemented the basic control function of the motor started, accelerated and decelerated in medium and low speed conditions. It will lay a good foundation for the development, transplantation and function expansion on different platforms for auto ECU control system.
\end{abstract}

\section{Introduction}

In recent years, as the booming of automotive electronic system, the major automobile manufacturers and parts suppliers launched their own ECU control system development platform, which leads to the motor control software reusability, difficult to maintain. In order to solve these problems, the big automobile manufacturers, auto parts suppliers and semiconductor both with software development companies of the world launched the automotive open system architecture (AUTOSAR) [1], whose purpose is to provide standardized, modular software architecture for automotive electronics software development. ECU motor control software research and design based on the software development method of AUTOSAR standard will become the future of motor driven software development trend of electric vehicle drive.

This paper ,based on the electric vehicle permanent magnet synchronous motor as control object, control method is the magnetic field vector oriented control[2], using Infineon TC1767 processor[3] as the ECU software development platform, has designed of the motor control ECU control system based on AUTOSAR standard. The bench test results show that, when the motor starting, accelerating controls and the decelerating, the motor ECU control system design can realize the control of permanent magnet synchronous motor well.

\section{The brief of AUTOSAR}

AUTOSAR uses a hierarchical, modular architecture, which can be divide into Application Layer, AUTOSAR Run Time Environment (RET) and Basic Software Layer (BSW), it can be shown in Figure 1.

The application layer of the AUTOSAR is made up by software components (SWC), which is connected by the virtual function bus[4](VFB). Every SWC has one or more than ports, it is the interfaces connect different SWC that can describe the detail communication content. Interface is divided into S-R(Sender-Receiver Interface) and C-S(Client-Server Interface). RTE is the core of AUTOSAR architecture, through which VFB achieves its specific function. Basic software layer is the standardized software layer, which provides the necessary services to software components. It is 
divided into three layers down: the service layer, ECU abstraction layer and microcontroller abstraction layer [5].

\section{Design of ECU motor control system}

According to AUTOSAR hierarchical, modular thinking, the general framework of ECU motor control system based on AUTOSAR architecture is shown in figure 2.

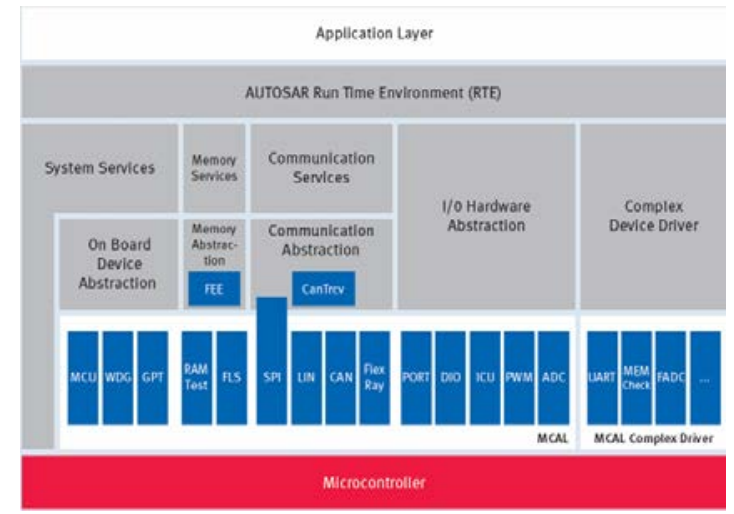

Fig.1 AUTOSAR software architecture

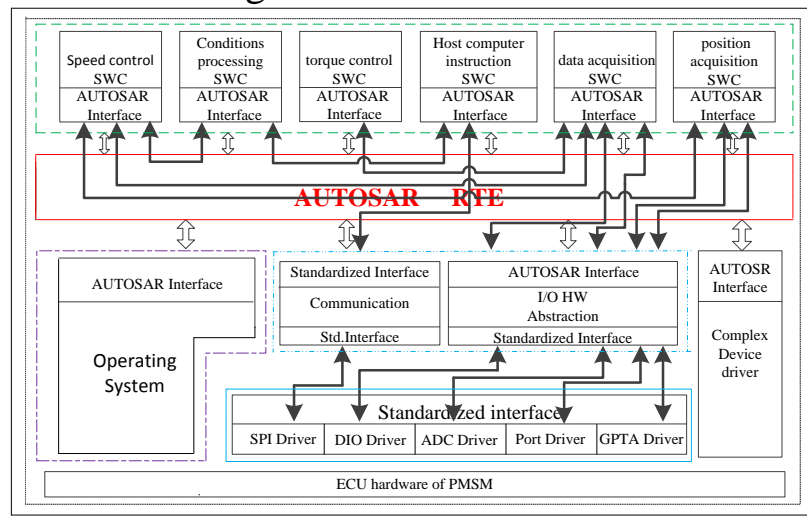

Fig.2 ECU motor control system architecture Based on AUTOSAR

Design of the application layer. The application layer is the main function of ECU motor control system, which is mainly composed of various functional software components. In this layer are designed six SWC according to the function of ECU control system, including speed control , torque control, condition processing, host computer instruction, data collection and position acquisition.

Speed control SWC and torque control SWC associated with motor control strategy, their mainly to complete FOC motor control algorithm. The host computer instruction SWC completed the command control to the motor, including the motor starting, speed control, braking and control mode settings. Data acquisition SWC and position acquisition SWC, which defines some interfaces access I/O hardware abstraction layer. It got motor three-phase stator current through HALL current sensor collected, got the stator three-phase winding temperature by temperature sensor acquisitioned, got the motor rotor angular position, mechanical angular position and angular velocity by rotating transformer from these interfaces.

Because of the limited space, the following detailed design of torque control SWC. It has six input interfaces, two output interfaces. These interfaces transmitted data elements, which designed to Sender-Receive type. All interfaces define of torque control software components such as table 1.

Tab.1 the define of torque control

\begin{tabular}{cccc}
\hline Interface name & Interfacetype & Date type & Date description \\
\hline $\mathrm{I}_{\mathrm{q}}$ ref & $\mathrm{S}-\mathrm{R}$ & float & The reference currentcalculationofq-axis \\
Start, Stop & $\mathrm{S}-\mathrm{R}$ & bool & The state of motor starting and stopping \\
$\mathrm{I}_{\mathrm{a}}, \mathrm{I}_{\mathrm{b}}$ & $\mathrm{S}-\mathrm{R}$ & float & The motor rotor electrical angle \\
Electrical angle & $\mathrm{S}-\mathrm{R}$ & Signed int & The motor rotor electrical angle \\
$\mathrm{K}_{\mathrm{p}} 、 \mathrm{~K}_{\mathrm{i}}$ & $\mathrm{S}-\mathrm{R}$ & float & The parameters of speed loop PI \\
Mode & $\mathrm{S}-\mathrm{R}$ & bool & Operating mode of the motor \\
PWM pattern & S-R & bool & FOC output PWM signal \\
\hline
\end{tabular}

Torque control software component is actually double closed current loop, which is first completed to coordinate transforming of the motor three-phase current, then d-axis and q-axis reference current used as input, through the PI controller, PARK inverse transform, SVPWM module generated 6 PWM waves drove inverter. It need to achieve the algorithm design, including PI 
controller of current loop, Park transform, Clarke transform in torque control SWC. Design torque control of PI controller is shown in figure3.

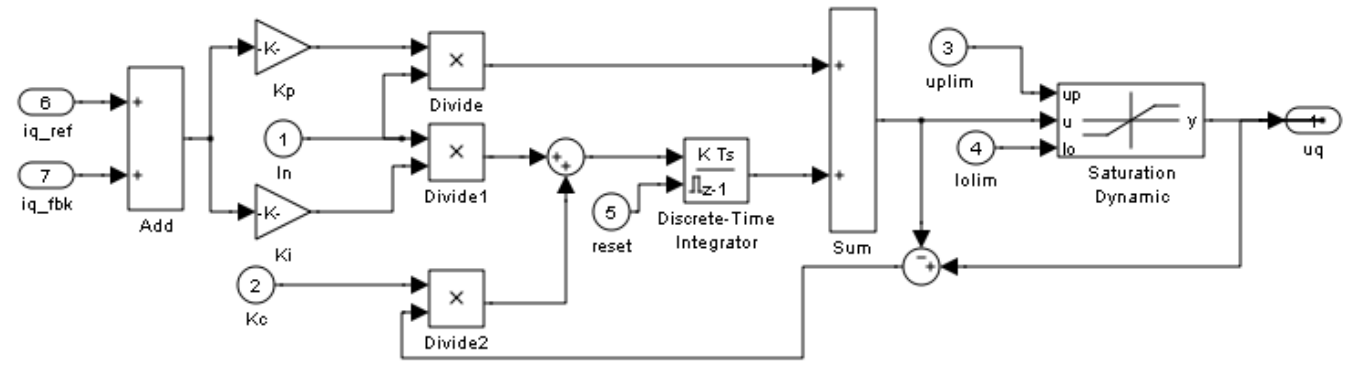

Fig.3 The torque loop of PI controller

Deign of VFB and RTE layer. RTE is the core of the AUTOSAR architecture, is the realization of VFB. The communication among software components is called by RTE API function instead of achieving directly, is managed and controlled by RTE, each API follow the same naming rules and only follow with software component self-description. To complete communication among software components such as speed control, torque control, host computer instruction, data acquisition and position acquire, also completed the communication among data acquisition SWC, position acquisition SWC and BSW in this paper.

Deign of ECU basic software layer. It is mainly to design of microcontroller abstraction layer and I/O hardware abstraction layer in the basic software layer. Microcontroller abstraction layer contains various modules driver, such as Port drive, DIO drive, ADC drive, GPTA drive, SPI drive. It provides a unified interface function of API, makes the upper software to independent of the microcontroller. I/O hardware abstraction layer to complete all the input and output signal acquisition, processing and abstract, it accesses various peripherals by microcontroller abstraction layer provides hardware driver, then provides access AUTOSAR interface to application layer software components.

\section{Result and Discussion}

In order to verify the feasibility of motor ECU control system based on AUTOSAR, the bench experiment on the control of PMSM was designed. The rated power of PMSM is 10KW, which was used the experimental motor in the motor drive control system. Other main parameters is listed as follows including six pair of pole number, rated voltage $109 \sim 190 \mathrm{~V}$, stator resistance $0.4 \Omega$, stator inductance $1.55 \mathrm{mH}$. To set PI controller parameters as follows speed loop $K_{p}=25, K_{i}=20$, current loop $i_{d}, K_{p}=0.018, K_{i}=25$, current loop $i_{q}, K_{p}=0.018, K_{i}=25$.

It set target motor speed to 600 $/ \mathrm{min}$ in PMSM low speed when test started, the measure response curve are shown in figure4. The motor started with good effect, can accelerate to 600 $/ \mathrm{min}$ within 0.6 second from the response curve. After the motor starts stability, the motor accelerated to $1000 \mathrm{r} / \mathrm{min}$ based on $600 \mathrm{r} / \mathrm{min}$, and adjusted the motor speed to $1500 \mathrm{r} / \mathrm{min}$, get the response curve shown in Figure 5. It can see the system has good ability to adjust the acceleration from the response curve. It did deceleration experiment again after acceleration, the motor deceased to $500 \mathrm{r} / \mathrm{min}$ based on $1000 \mathrm{r} / \mathrm{min}$, get the response curve shown in Figure 6 . It can see the system has good ability to adjust the deceleration from the response curve.

\section{Conclusions}

This paper, to design ECU motor control system adopted the modularization idea, including ECU application layer, RTE layer and basic software layer. The experimental bench showed that based on AUTOSAR completed implemented the basic control function of the motor started, accelerated and decelerated. From the measured curve can see that the system has good speed regulation ability. The Method based on the standard of AUTOSAR improves the flexibility, configurability and reusability 
of the code of the system, which were used to improved the driving motor control system development efficiency on electric vehicle.
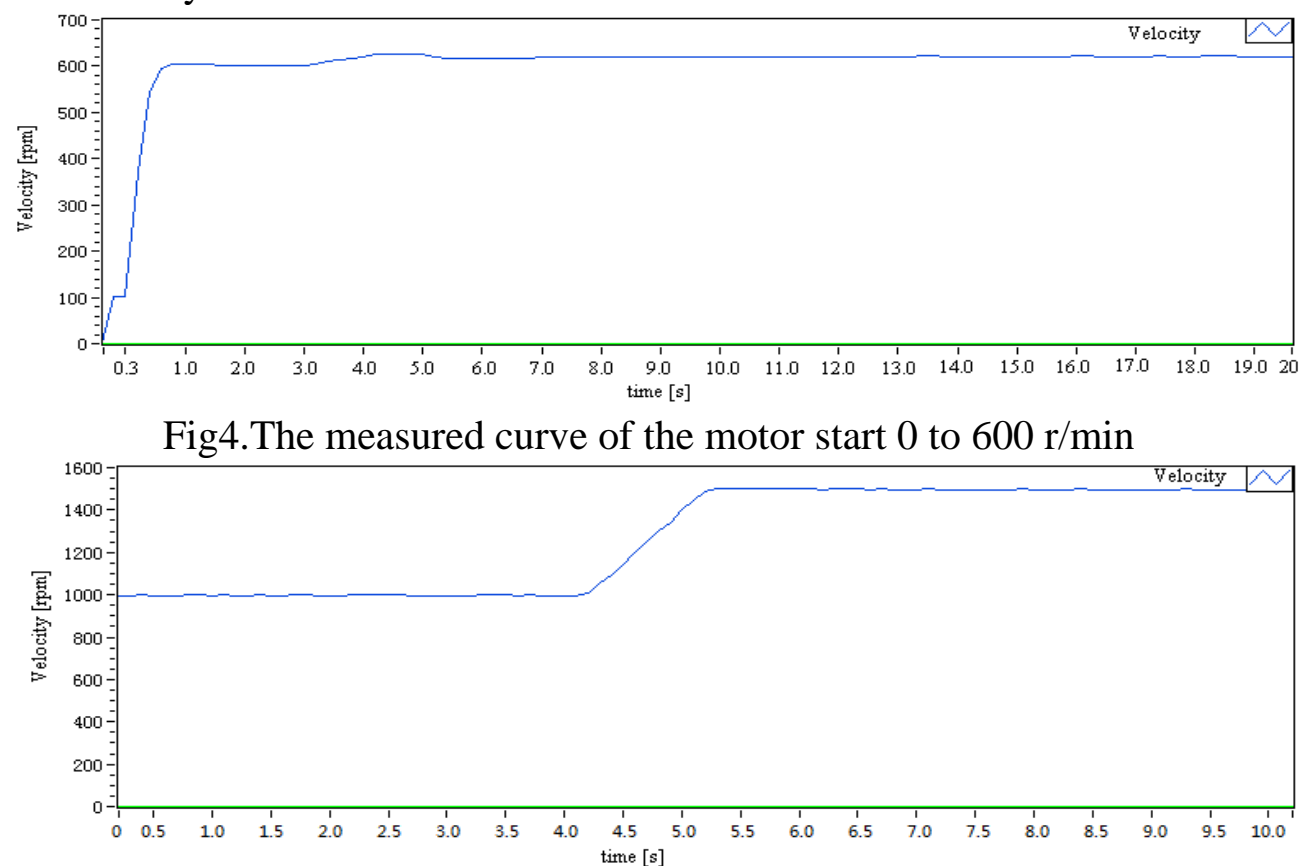

Fig5. The measured cure with rotate speed $1000 \mathrm{r} / \mathrm{min}$ increasing to $1500 \mathrm{r} / \mathrm{min}$

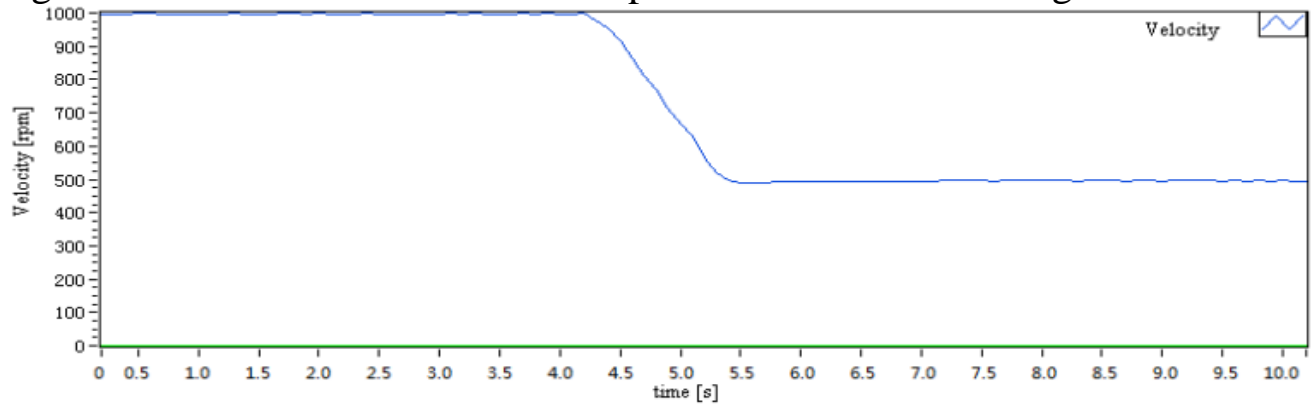

Fig6. The measured cure with rotate speed1000r/min reducing to 500r/min

\section{Acknowledgement}

This research was financially supported by the key technology of pure EV motor control and drive system research and design (cstc2014yykfA4004).

\section{References}

[1] Infineon controller. The AUTOSAR software of Infineon [OL]. (2009-3-25) http://www. infineon.com/cms/cn/product/Microcontrollers/.

[2] Huangzhi-yu, Zhangxiao-lei, Zhengtai-xiong. Realization of Permanent Magnet Synchronous Motor Control System Based on FOC for Electric Vehicle Drive[J]. ELECTRIC DRIVE, 2013,43(12):7-11.

[3] TC1767 32-bit Single-Chip Microcontroller user’s Manual_V1.1 [OL]. (2009-05) http:// www.infineon.com.

[4] HuQi, LiHong, Zhaoming-de. Design and implementation of ECU software for electronic gasoline engine based on AUTOSAR[J]. Journal of Zhejiang university: Engineering and Technology, 2011, 45(6): 1119-1123.

[5] Zhangpei-feng. Gasoline Engine ECU Software Design according on AUTOSAR[D]. Huangzhou: Zhejiang university,2010. 